BULL. AUSTRAL. MATH. SOC.

MOS 06A35, 16A66, 16A78

VOL. $5(1971), 423-424$.

\title{
Abelian Rickart semirings
}

\author{
William H. Cornish
}

An abelian Rickart semiring is a semiring with 0,1 and non-commutative multiplication in which the (left) annihilator of each element is a direct summand. They generalize commutative Rickart rings and Stone lattices and form a well-behaved class of reduced semirings.

In Chapter 1 these semirings are defined equationally. A subdirect representation is given. The result is not typical of reduced semirings, see [1]. The relationship between homomorphisms and minimal prime ideals is obtained and used to study prime ideals with the hull-kernel topology. If the left ore condition holds then a ring is abelian Rickart iff it has the same idempotents as its classical ring of quotients which is itself strongly regular. Consequently, there are few, if any, injective commutative Rickart rings. Details appear in [2].

Kist's characterization of minimal prime ideals is extended to non-commutative reduced semigroups in Chapter 2. Applying this to rings and f-rings yields a characterization of the Rickart ring of continuous bounded integer-valued functions as a non-commutative lattice-ordered ring.

Chapter 3 studies reduced semirings satisfying the double annihilator condition, that is, for each $x$ there is an $x^{\prime}$ such that ann ann $x$ = ann $x^{\prime}$. Using minimal prime ideals, many characteristic properties are presented and for rings the condition is equivalent to possessing a strongly regular ring of left quotients. Via these results a new development of Goldie's Theorem is given for the case of reduced rings.

Chapter 4 deals with semirings in which each prime contains a unique minimal prime and abelian Rickart semirings are characterized amongst them as semirings satisfying the double annihilator condition. More specialized

Received 2 August 1971. Thesis submitted to The Flinders University of South Australia, December 1970. Degree approved, June 1971.

Supervisor: Professor B. Abrahamson. 
results on lattices are also given, see [3] and [4].

\section{References}

[1] William H. Cornish, "Subdirectly irreducible semirings and semigroups without non-zero nilpotents", Canad. Math. Bull. (to appear).

[2] William H. Cornish, "The variety of commutative Rickart rings", Nanta Math. (to appear).

[3] William H. Cornish, "Normal lattices", J. Austral. Math. Soc. (to appear).

[4] William H. Cornish, "Annulets and $\alpha$-ideals in a distributive lattice", J. Austral. Math. Soc. (to appear). 